women and women with a high parity who are exposed to a relatively high risk of twin pregnancy make increasing use of contraceptives and abortion which is being liberalized in the GDR since I972.

Twin pregnancy represents a high risk to the survival of fetuses since insufficient nutrition frequently results in hypotrophic development and low birth weight. The frequency of the occurrence of low birth weight in children from a multiple birth is Io times higher than in all newborn children and the early neonatal mortality of children from a multiple birth is 8 times higher than that of all newborn infants.

The disproportion between the placenta and the fetuses which we usually find in multiple pregnancies may also result in the occurrence of cerebral damage. Cerebral damage is 30 times higher in children of low birth weight than in children of normal birth weight. In the period 1965-1967 perinatal death associated with cerebral damage (recorded on the death certificate) accounted for $5.7 \%$ of all children of low birth weight and for $0.2 \%$ of children weighing at birth over 2500 g. Cerebral damage in the surviving twin children puts a heavy burden on the child, the family, and society. Early diagnosis of multiple pregnancy and early in-patient care of women with multiple pregnancy will improve the development of multiple fetuses and give children from a multiple birth much better chances in life. In all twin studies it has to be considered that a relatively high proportion of twins is affected to a higher or lesser degree by cerebral damage.

Dr. I. Leetz, Akademie für Artzliche Fortbildung, Noldnerstrasse $34-36$, I I 34 Berlin, German Democratic Republic

\section{DURATION AND COMPLICATIONS OF MULTIPLE PREGNANGIES}

\section{BOLOGNESI}

The Gregor Mendel Institute of Medical Genetics and Twin Research, Rome, Italy

Multiple pregnancies reach full term only in a remarkably lower percentage with respect to single pregnancies. Even in the case of multiparae, delivery occurs some three weeks before term.
Complications may concern either pregnancy and puerperium or delivery. The former include ( $I$ ) abortions, especially frequent in the case of monoplacental and monochorial pregnancies; (2) rarer events, such as hydramnios, placenta praevia, association of an ectopic pregnancy with an intrauterine pregnancy, molar degeneration of one egg with normal development of the other; (3) gestoses of the first and second trimester, more frequent than in single pregnancy; and (4) higher incidence, during puerperium, of hemorrhage, phlebitis, and uterine subinvolution.

With respect to delivery, normal delivery only occurs in approximately $70 \%$ of twin pregnancies, the period of dilatation being generally longer than normal. Better assistance and techniques during labor and especially in the interval between delivery of first and second twin have remarkably reduced the higher mortality rate, especially for the second twin.

Prof. M. Bolognesi, Istituto Mendel, Piazza Galeno 5, oor6r Roma, Italy

\section{INTRAUTERINE HYPOXIA - A PHENOMENON PROPER TO THE SECOND TWIN}

\section{R. DEROM, M. THIERY}

Department of Obstetrics, State University, Ghent, Belgium

During a 5-year period twin births occurring at the university hospital were investigated for the presence of clinical and biochemical symptoms of intrauterine hypoxia. It appears that intrauterine hypoxia, when detectable, is limited to the second twin. Some factors which may influence the oxygenation of the second twin have been analysed, i.e., the mode of delivery, the time interval between deliveries, and the duration of gestation. No single explanation for the genesis of the hypoxia seems to be valid.

Prof. R. Derom, Department of Obstetrics, De Pintelaan 136 , B-9ooo Ghent, Belgium 